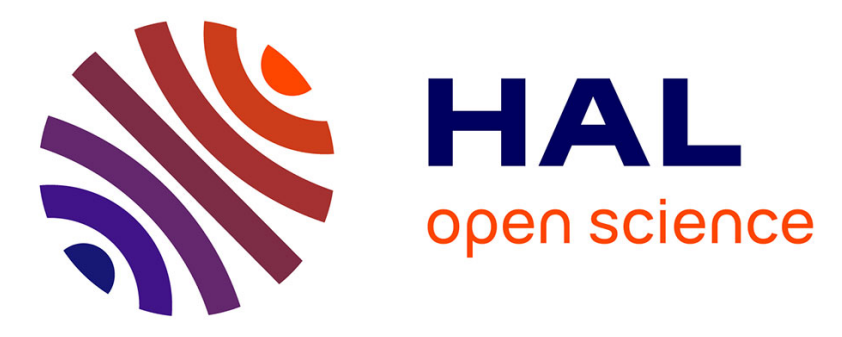

\title{
Electron-phonon coupling and charge-transfer excitations in organic systems from many-body perturbation theory
}

Carina Faber, Ivan Duchemin, Thierry Deutsch, Claudio Attaccalite, Valerio Olevano, Xavier Blase

\section{To cite this version:}

Carina Faber, Ivan Duchemin, Thierry Deutsch, Claudio Attaccalite, Valerio Olevano, et al.. Electronphonon coupling and charge-transfer excitations in organic systems from many-body perturbation theory. Journal of Materials Science, 2012, 47 (21), pp.7472. 10.1007/s10853-012-6401-7 . hal00730389

\section{HAL Id: hal-00730389 \\ https://hal.science/hal-00730389}

Submitted on 10 Sep 2012

HAL is a multi-disciplinary open access archive for the deposit and dissemination of scientific research documents, whether they are published or not. The documents may come from teaching and research institutions in France or abroad, or from public or private research centers.
L'archive ouverte pluridisciplinaire HAL, est destinée au dépôt et à la diffusion de documents scientifiques de niveau recherche, publiés ou non, émanant des établissements d'enseignement et de recherche français ou étrangers, des laboratoires publics ou privés. 


\title{
Electron-phonon coupling and charge-transfer excitations in organic systems from many-body perturbation theory \\ The Fiesta code, an efficient Gaussian-basis implementation of the $G W$ and Bethe-Salpeter formalisms
}

\author{
Carina Faber • Ivan Duchemin • Thierry Deutsch • Claudio Attaccalite • \\ Valerio Olevano - Xavier Blase
}

Received: date / Accepted: date

\begin{abstract}
We review in the present article recent developments within the framework of $a b$ initio manybody perturbation theory aiming at providing an accurate description of the electronic and excitonic properties of $\pi$-conjugated organic systems currently used in organic photovoltaic cells. In particular, techniques such as the $G W$ and Bethe-Salpeter formalisms are being benchmarked for acenes, fullerenes, porphyrins, phthalocyanines and other molecules of interest for solar energy applications. It is shown that not only the electronic properties, but also the electron-phonon coupling matrix elements, and the charge-transfer excitations in donor/acceptor complexes, are accurately described. The present calculations on molecules containing up to a hundred atoms are based on a recently developed Gaussian auxiliary basis implementation of the $G W$ and Bethe-Salpeter formalism, including full dynamics with contour deformation techniques, as implemented in the FIESTA code.
\end{abstract}

Keywords Ab initio simulations · Many-body perturbation theory · Organic photovoltaics

\section{Introduction}

It is certainly difficult to anticipate the evolution of organic photovoltaic cells at an industrial level [1-4].

C. Faber, V. Olevano, C. Attaccalite, X. Blase

Institut Néel, CNRS/UJF, B.P. 166, 38042 Grenoble, Cedex 09, France.

E-mail: xavier.blase@grenoble.cnrs.fr

I. Duchemin, T. Deutsch

Laboratoire de Simulation Atomistique (L_Sim), SP2M, INAC, CEA-UJF 17 Av. des Martyrs, 38054 Grenoble, France.
Even though organic systems offer an extremely large flexibility in terms of available molecules and architectures, the rather low quantum efficiency (a few percent), as compared to standard silicon-based solar cells, and the problems of stability under UV radiations, are serious limitations that need to be addressed. It has been shown however that significant gains $[5,6]$ can be achieved by tuning the absorption spectrum, band gap and band offsets of the active molecules/polymers in standard donor/acceptor hetero-structure cells [7-9]. Using computer quantum simulations to predict such spectroscopic parameters for actual size molecules or polymers would therefore be of much interest.

Density functional theory (DFT) remains the method of choice for the theoretical study of structural and dynamical properties, a conclusion that has been recently consolidated by the implementation of van der Waals forces into standard exchange-correlation functionals, with applications to organic systems [10]. Nevertheless, it remains that DFT is a ground-state formalism with intrinsic difficulties to describe electronic excitation energies of the kind involved in photoemission experiments. While the so-called $\triangle \mathrm{SCF}$ techniques, relying on differences of total ground-state energies for accessing the frontier orbital energies in finite size systems, offer an excellent compromise between computer time and accuracy, difficulties exist when it comes to apply them to the full electronic energy spectrum and/or to infinite systems.

In these cases, one is bound to rely on the DFT Kohn-Sham eigenstates which cannot be identified to true quasiparticle states. In particular, the standard "band gap problem", documented since the mid-60s for extended semiconductors [11], is dramatically enhanced in absolute value in the case of organic molecules, with errors of the order of several $\mathrm{eV}$. For sake of illustration, 
the DFT-LDA and DFT-B3LYP Kohn-Sham HOMOLUMO gap for the $C_{60}$ fullerene are found to be $1.6 \mathrm{eV}$ and $2.8 \mathrm{eV}[12,13]$ to be compared to the $4.9-5.0 \mathrm{eV}$ experimental value [14]. Several attemps to cure such problems within the framework of DFT are currently undertaken, working on the exchange-correlation functionals, within e.g. the framework of range-separated [15] or orbital-dependent self-interaction corrected [16] functionals.

In this report, we present recent achievements within the framework of many-body perturbation theory for the $a b$ initio study of the electronic properties, electronphonon coupling and charge-transfer excitations, in organic molecules and donor/acceptor dyads of interest for photovoltaic applications, including fullerenes, porphyrins, phthalocyanines, and tetracyanoethylene acceptor combined with several benzene, naphthalene, and anthracene donor derivatives. We adopt in particular the $G W$ and Bethe-Salpeter (BSE) formalisms as implemented in a recently developed auxiliary Gaussianbasis approach with contour-deformation techniques, the FiEsta code. Our results show that an excellent agreement with experiment can be obtained provided that, beyond standard single-shot $G_{0} W_{0}$ based on DFTLDA eigenstates, one performs a simple self-consistency on the eigenvalues, or start from Hartree-Fock-like singleparticle energies.

\section{Methodology}

Beyond the well documented case of inorganic bulk semiconductors, the use of $G W[17-20]$ and BSE [21$25]$ formalisms for the study of organic systems remains relatively scarce, with early studies devoted e.g. to bulk periodic acenes [26-28] or PTCDA [29], as prototype organic semiconductors, and periodic polymers [30-33]. In another context, the importance of self-energy effects on the properties of organic molecules deposited on semiconducting or metallic surfaces, as a mean in particular to better understand molecular conductance properties, have been considered using several approaches, from model image-charge analysis, static (COHSEX) approximations, to full-fledged GW calculations [3440].

In the case of isolated (gas phase) molecules of interest for photovoltaic applications, such as fullerenes, porphyrins and phthalocyanines, the rather large number of atoms (several dozens), and the need to include a large amount of vaccuum to reduce the Coulomb interactions between periodic image cells in standard planewave implementations, certainly explains the limited number of studies undertaken by few groups worldwide $[37,41-49]$. Some of these studies were in partic- ular made possible by implementing techniques which allow to bypass the need of the empty states manifold [45], or by using localized basis, such as strictly localized bases [48], Wannier representations [45], or Gaussian bases $[43,46]$ of the type currently used in most quantum chemistry studies of molecular systems. Such real-space localized bases allow to reduce, for molecular systems, both the size of the dielectric matrices and number of conduction bands. Localized bases are in particular insensitive to the amount of vaccuum being added. The results described here below are based on a recent implementation of the $G W / \mathrm{BSE}$ formalism with localized-basis, the FIESTA package $[46,47,50-52]$, namely an auxiliary Gaussian basis implementation of the $G W$ and BSE formalisms, exploiting contour deformation techniques beyond plasmon pole models.

\subsection{The many-body $G W$ and Bethe-salpeter equations}

Central to the $G W[17-20]$ and BSE [21-25] formalisms stands the dynamically screened Coulomb potential $W(\omega)$ related to the bare Coulomb potential $v$ and the unscreened susceptibility $\chi^{0}$ through a Dyson-like equation:

$$
[W(\omega)]=[v]+[v] S^{-1}\left[\chi^{0}(\omega)\right] S^{-1}[W(\omega)],
$$

where the notations $[f]$ stands for the matrix representation of any two-point function $f\left(\mathbf{r}, \mathbf{r}^{\prime}\right)$ in the chosen auxiliary-basis, with corresponding overlap $S$ matrix. The susceptibility $\chi^{0}(\omega)$ is traditionaly calculated from input Kohn-Sham DFT one-body eigenstates $\left(\varepsilon_{i}, \phi_{i}\right)$ :

$\left[\chi^{0}(\omega)\right]_{\mu, \nu}=\sum_{i, j}\left(f_{i}-f_{j}\right) \frac{<\phi_{i}|\mu| \phi_{j}><\phi_{j}|\nu| \phi_{i}>}{\omega+\varepsilon_{i}-\varepsilon_{j}+i 0^{+}}$

with $\left(f_{i}\right)$ the occupation factors. The calculated screened Coulomb potential $W(\omega)$ allows to build the non-local and energy dependent self-energy operator $\Sigma$, which accounts for exchange and correlation in the present quasiparticle formalism [17] and reads:

$$
\begin{aligned}
\Sigma^{G W}\left(\mathbf{r}, \mathbf{r}^{\prime} \mid \mathbf{E}\right) & =\frac{i}{2 \pi} \int d \omega e^{i 0^{+} \omega} G\left(\mathbf{r}, \mathbf{r}^{\prime} \mid E+\omega\right) W\left(\mathbf{r}, \mathbf{r}^{\prime} \mid \omega\right) \\
G\left(\mathbf{r}, \mathbf{r}^{\prime} \mid \omega\right) & =\sum_{n} \phi_{n}(\mathbf{r}) \phi_{n}^{*}\left(\mathbf{r}^{\prime}\right) /\left(\omega-\varepsilon_{n} \pm i \delta\right)
\end{aligned}
$$

where the single-particle time-ordered Green's function $G$ is again built from the $\left(\varepsilon_{i}, \phi_{i}\right)$ eigenstates. The selfenergy correction to the input DFT Kohn-Sham eigenvalues requires the calculation of the self-energy at the quasiparticle energy $\varepsilon_{n}^{Q P}$, namely: 


$$
\varepsilon_{n}^{Q P}=\varepsilon_{n}+<\phi_{n}\left|\Sigma^{G W}\left(\varepsilon_{n}^{Q P}\right)-V_{x c}^{D F T}\right| \phi_{n}>
$$

The $\varepsilon_{n}^{Q P}$ represents well-defined charged excitation energies of the kind obtained in a direct or inverse photoemission experiment and represent thus proper electronic energy levels.

Optical properties require the knowledge of neutral excitations upon photon absorption. As such, both the promoted electron and "left-behind" hole are simultaneously present in the system and can interact. As shown below, in the case of organic systems, due to confinement and poor screening, this interaction can be very large, so that the optical gap is significantly smaller than the quasiparticle band gap. In the present formalism, the electron-hole (excitonic) interaction is accounted for by the Bethe-Salpeter equations (BSE). Namely, the neutral excitation energies can be obtained as the eigenvalues of the Bethe-Salpeter $H^{e-h}$ Hamiltonian [21-25] which, in the $\phi_{i}^{e}(r) \phi_{j}^{h}\left(r^{\prime}\right)$ product basis of the non-interacting unoccupied $\phi_{i}^{e}$ and occupied $\phi_{j}^{h}$ single-particle states, is composed of three terms as follows:

$$
\begin{aligned}
H_{i j, k l}^{\text {diag }} & =\delta_{i, k} \delta_{j, l}\left(\varepsilon_{i}^{Q P}-\varepsilon_{j}^{Q P}\right) \\
H_{i j, k l}^{\text {direct }} & =-\int d \mathbf{r} d \mathbf{r}^{\prime} \phi_{i}^{e}(\mathbf{r}) \phi_{j}^{h}\left(\mathbf{r}^{\prime}\right) W\left(\mathbf{r}, \mathbf{r}^{\prime}\right) \phi_{k}^{e}(\mathbf{r}) \phi_{l}^{h}\left(\mathbf{r}^{\prime}\right) \\
H_{i j, k l}^{\text {exchange }} & =2 \int d \mathbf{r} d \mathbf{r}^{\prime} \phi_{i}^{e}(\mathbf{r}) \phi_{j}^{h}\left(\mathbf{r}^{\prime}\right) v\left(\mathbf{r}, \mathbf{r}^{\prime}\right) \phi_{k}^{e}\left(\mathbf{r}^{\prime}\right) \phi_{l}^{h}(\mathbf{r})
\end{aligned}
$$

It is important to note that in the $H^{\text {direct }}$ term, the two electron (or hole) eigenstates are taken to be at the same position in space, in contrast with the exchange $H^{\text {exchange }}$ term. The expression of the optical excitations in the $\mid \phi_{i}^{e} \phi_{j}^{h}>$ basis is reminiscent of the Casida's equations for time-dependent DFT (TDDFT) $[54,55]$ with the crucial differences that in TDDFT, (a) the "non-interacting" diagonal part is built from the Kohn-Sham DFT eigenstates (not the $G W$ quasiparticle energies) and (b) the electron-hole interaction is accounted for by matrix elements, in the product basis, of the bare Coulomb potential $v$, complemented by the exchange-correlation kernel $f^{x c}$, namely, with $K^{x c}\left(r, r^{\prime}\right)=v\left(r, r^{\prime}\right)+f^{x c}\left(r, r^{\prime}\right)$ :

$$
\left[K^{x c}\right]_{i j, k l}=\int d \mathbf{r} d \mathbf{r}^{\prime} \phi_{i}^{e}(\mathbf{r}) \phi_{j}^{h}(\mathbf{r}) K^{x c}\left(\mathbf{r}, \mathbf{r}^{\prime}\right) \phi_{k}^{e}\left(\mathbf{r}^{\prime}\right) \phi_{l}^{h}\left(\mathbf{r}^{\prime}\right) .
$$

where the adiabatic approximation has been assumed for $f^{x c}$. Of crucial importance in the case of charge transfer excitations, when hole and electron states are spatially separated, we observe that the $\phi_{i}^{e}(\mathbf{r}) \phi_{j}^{h}(\mathbf{r}) \operatorname{prod}-$ uct, that appears in the TDDFT matrix elements, go to (a)

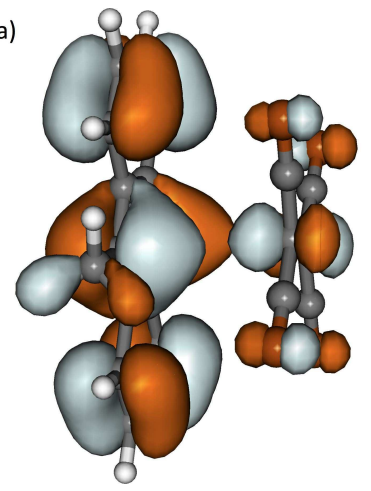

(b)

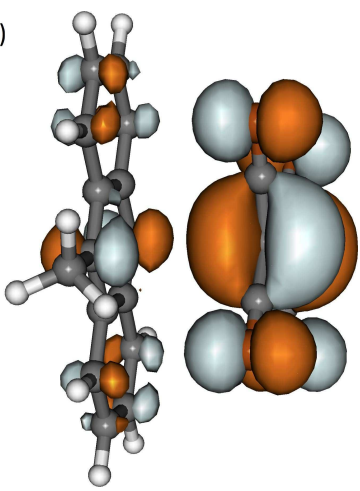

Fig. 1 (Color online) Isocontour representation of the (a) highest-occupied (HOMO), and (b) lowest unoccupied (LUMO) molecular orbitals of the tetracyanoethylene with the 9-methyl-anthracene complex. Contours at $\pm 15 \%$ of the maximum charge density. Orbitals obtained at the DFT-LDA TZ2P level. The (partial) charge-transfer character of the HOMO to LUMO excitation is apparent (see Section 4).

zero, so that one is left with the non-interacting diagonal part, namely the difference between DFT KohnSham unoccupied and occupied states. In that case, the charge-transfer excitation energy is just the donorHOMO to acceptor-LUMO DFT Kohn-Sham gap without electron-hole interaction. This is not the case in the BSE formalism since, in the direct term, the hole and electron states are not taken at the same spatial position and are connected by the non-local $W\left(\mathbf{r}, \mathbf{r}^{\prime}\right)$ potential. These observations underline the complexity of finding a proper kernel within TDDFT that would "compensate" for the vanishingly small value of the wavefunction products in the case of charge-transfer excitations. A typical donor/acceptor complex yielding charge transfer excitations is represented in Fig. 1.

\subsection{The Fiesta code: a Gaussian basis implementation}

Building on previous initiatives to formulate the MBPT $G W$ and BSE formalisms on auxiliary Gaussian bases [30,56-58], arguably better suited to molecular systems than the standard planewave implementations, the FIESTA code [46] expresses the non-local two-body operators such as the susceptibilities, bare or screened Coulomb potentials, and self-energies, on an even-tempered [59] auxiliary Gaussian basis. With Kohn-Sham states originating from pseudopotential calculations, the standard bases available from all-electron quantum chemistry studies cannot be straightforwardly imported, since the auxiliary basis must project on the Hilbert space generated by the product of unoccupied and occupied states (the so-called product space $[48,60,61]$ ). As such, 


\begin{tabular}{|l|c|c|c|c|c|c|}
\hline KS basis & \multicolumn{2}{|c|}{ pentacene } & \multicolumn{2}{c|}{$\mathrm{H}_{2} \mathrm{P}$} & \multicolumn{2}{c|}{$\mathrm{C}_{60}$} \\
\hline & IE & gap & IE & gap & IE & gap \\
\hline LDA (DZP) & 4.94 & 1.10 & 5.64 & 1.94 & 6.37 & 1.58 \\
\hline$G_{0} W_{0}(\mathrm{DZP})$ & 5.98 & 4.21 & 6.55 & 4.79 & 7.28 & 4.44 \\
\hline$G W_{p s c}(\mathrm{DZP})$ & 6.12 & 4.76 & 6.70 & 5.31 & 7.41 & 4.76 \\
\hline$G W_{p s c}(\mathrm{TZP})$ & 6.20 & 4.68 & 6.70 & 5.25 & 7.42 & 4.70 \\
\hline$G W_{p s c}(\mathrm{TZ2P})$ & 6.26 & 4.67 & 6.76 & 5.23 & 7.47 & 4.67 \\
\hline Exp. & 6.6 & 5.2 & 6.9 & - & 7.6 & 4.9 \\
\hline
\end{tabular}

Table 1 Evolution of the $G W_{p s c}$ ionization (IE) and band gap energies of selected molecules as a function of the input Kohn-Sham (KS) basis size. DZP, TZP and TZ2P stand for double-zeta plus polarization, triple-zeta plus polarization, and triple-zeta plus double polarization. The index (psc) stands for partial self-consistency on the eigenvalues (see below). The LDA, $G_{0} W_{0}(\mathrm{LDA})$ and $G W_{p s c}$ calculations with the DZP basis are from Ref. [46]. Results are in $\mathrm{eV}$.

auxiliary bases in the framework of many-body perturbation theory still needs careful testing for chemical species all across the periodic table. This was done in some details in Refs. $[46,47,50]$ for a large range of organic molecules such as fullerenes, porphyrins, phthalocyanines, DNA/RNA nucleobases, and charge-transfer complexes for which careful comparisons with planewave calculations have been performed (see Section 4 here below). By comparison with larger bases, it was shown in particular that four Gaussians with proper localization coefficients for each $s, p$, and $d$ channels were sufficient for convergency within a tenth of an $\mathrm{eV}$ (see Table I of Ref. [46]). For first row elements, the influence of $f$ channels was found to be negligible.

In the present implementation, we use the KohnSham eigenstates generated by the DFT SIESTA package [53] which is a pseudopotential DFT code relying on strictly localized orbital bases [62]. The size of the DFT basis controls the quality of the Kohn-Sham states and the number of conduction bands. For sake of illustration, the number of conduction bands per carbon atom amounts to 11,15 and 20 with a double-zeta plus polarisation (DZP) basis, a triple-zeta plus polarisation (TZP) basis, and a large triple-zeta plus double polarisation (TZ2P) basis, respectively. While the DZP basis is probably the minimal basis that should be used for the study of excited state properties, the comparison with TZP and TZ2P calculations (Table 1) for pentacene, free-base porphyrine $\left(\mathrm{H}_{2} \mathrm{P}\right)$ and $C_{60}$ shows that the ionization energy (IE) and gap are already quite well converged, within $0.1-0.2 \mathrm{eV}$, with a tendency to an increase/decrease of the IE/gap with larger bases. Such a discrepancy between DZP and larger bases calculations can be compared to the magnitude of the $G W$ correction to DFT-LDA values, which is of several eV.

Contrary to previous implementation [56], we do not use any plasmon pole approximation for extending the screened Coulomb potential to finite frequencies. We use contour deformation techniques with an integration along the imaginary axis complemented by the evaluation of the residual of the poles in the first and third quadrant for states away from the band edges [20,63]. The divergency for $E \rightarrow \varepsilon_{n}$ and $\omega \rightarrow 0$ of the integrand in the imaginary axis integration contribution to the correlation energy:

$$
I_{n}(E)=-\int_{0}^{+\infty} \frac{d \omega}{\pi} \frac{E-\varepsilon_{n}}{\left(E-\varepsilon_{n}\right)^{2}+\omega^{2}} \tilde{W}_{n}(i \omega)
$$

with $\quad \tilde{W}_{n}(i \omega)=<\phi_{n}\left|W\left(\mathbf{r}, \mathbf{r}^{\prime} \mid i \omega\right)-v\left(\mathbf{r}, \mathbf{r}^{\prime}\right)\right| \phi_{n}>$, can be taken care of by adding/subtracting an analytically integrable function with similar behavior in the $\omega \rightarrow$ 0 limit. By further folding the $[0,+\infty]$ energy range onto $[0,1]$ by a change of variable, typically 12 special (Gaussian weighted) imaginary frequencies are needed for the integration along the imaginary axis (see Ref. [46] for more details).

With the above described implementation, the IE and HOMO-LUMO gap of 13 molecules of interest for photovoltaic applications were studied in Ref. [46]. Results concerning the IEs are summarized in Fig. 2. As expected, the IEs and gaps are dramatically improved within the standard single-shot $G_{0} W_{0}$ (LDA) calculations starting from input DFT-LDA Kohn-Sham eigenstates. The mean absolute error (MAE) was found to be significantly reduced, from $1.83 \mathrm{eV}$ to $0.47 \mathrm{eV}$ for the ionization energy, and from $4.10 \mathrm{eV}$ to $0.76 \mathrm{eV}$ for the energy gap, respectively, as compared to experiment.

Even though very satisfactory, such an improvement still leaves a noticeable discrepancy as compared to experiment, with in particular a $0.76 \mathrm{eV}$ MAE on the HOMO/LUMO gap. The limitations of the standard $G_{0} W_{0}$ (LDA) calculations for molecular systems have been recently discussed by several authors $[46,47,50$, $64,65,67]$. As emphasized above, the DFT-LDA starting point is extremely inaccurate, and probably not the best zeroth order framework to start a perturbation theory. As shown below in section 4, not only the $G_{0} W_{0}$ (LDA) quasiparticle gap, but also the resulting excitation energies within the subsequent $B S E$ framework, are still too small as compared to experiment [47]. A large variety of solutions were proposed, starting from a very simple diagonal Hartree-Fock-like scheme $[46,64]$, where the DFT exchange-correlation contribution to the Kohn-Sham eigenstates is just replaced, in the $G_{0} W_{0}$ spirit, by the bare exchange $\Sigma^{X}$ contribution within the LDA basis: $\varepsilon_{n}^{H F} \approx \varepsilon_{n}^{L D A}+<\phi_{n}^{L D A} \mid \Sigma^{X}-$ $V^{x c} \mid \phi_{n}^{L D A}>$, to starting Kohn-Sham states obtained with hybrid functionals [49], and finally much more computationally intensive full self-consistent approaches where eigenvalues and eigenstates are updated [65,67]. 


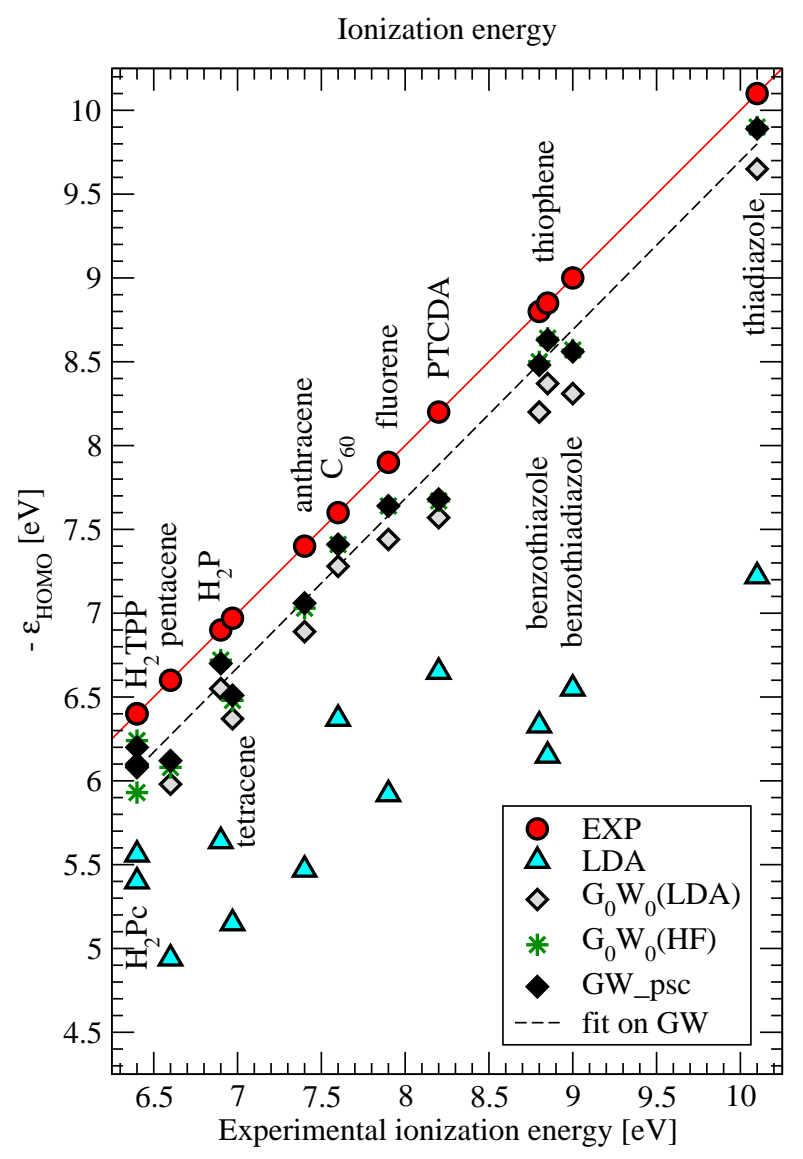

Fig. 2 (Color online) Experimental and theoretical energies in eV. Red circles: experimental values; light blue triangles up: LDA Kohn-Sham HOMO energy; grey diamonds: nonself-consistent $G_{0} W_{0}$ (LDA) value; black diamonds: $G W_{p s c}$ value with self-consistency on the eigenvalues; green stars: non self-consistent $G_{0} W_{0}$ (HF) (see text). The black dashed line is a least-squares fit of the $G W_{p s c}$ results. The figure has been formatted so as to preserve the same physical scale on both axes. Adapted from Ref. [46].

As shown in Fig. 2, in Tables 1 and 4, and in Refs. [46, $47,50]$, the Hartree-Fock ansatz, or a simple partial selfconsistency on the eigenvalues only, labeled $G W_{p s c}$ here below, already significantly improve on the $G_{0} W_{0}(\mathrm{LDA})$ results, increasing the HOMO-LUMO gap by $0.5-0.6 \mathrm{eV}$ as compared to $G_{0} W_{0}(\mathrm{LDA})$ results for acenes, fullerenes, porphyrins, phthalocyanines [46], DNA/RNA nucleobases [47], and a similar opening for the low lying excitation energies in donor/acceptor dyads [47]. Such an opening significantly improves the agreement with experiment.

Besides the value of the ionization energy and band gap, a final observation concerning the quasiparticle eigenstates in organic systems is that the $G W$ correction has been shown to potentially change the ordering of the states close to the gap as compared to DFT-LDA calculations. An important case in the family of organic systems, even though bringing us somehow away (a)

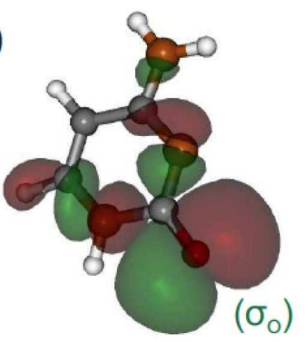

DFT-LDA

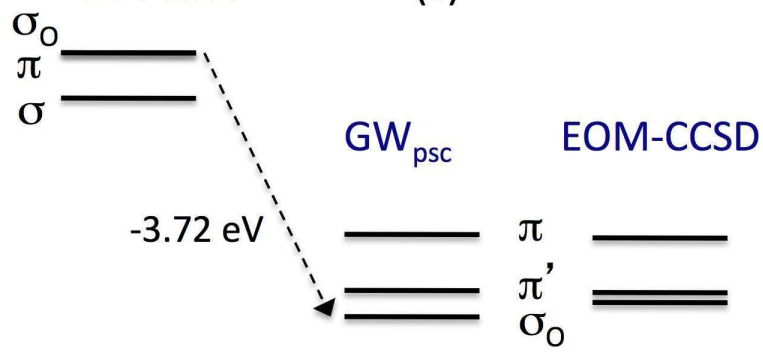

(b)

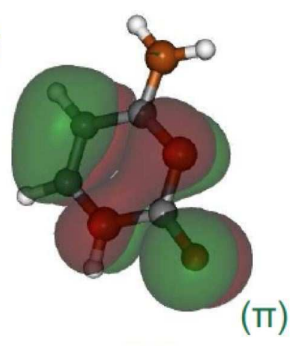

(c)
Fig. 3 (Color online) (a) and (b) Isodensity surface plot of the two highest occupied state of cytozine within DFT-LDA. (c) Three highest occupied energy levels of cytozine within DFT-LDA and $G W_{p s c}$, from Ref. [50], and a many-body coupled-cluster equation-of-motion technique (EOM-CCSD) from Ref. [68].

from photovoltaic systems, is that of DNA/RNA nucleobases, where the difference of energy between the occupied out-of-plane $\pi$-states and in-plane $\sigma$-states can be affected by more than an $\mathrm{eV}$ [50], ruling out the naïve interpretation of the $G W$ correction as a simple gap opening "scissor operator". This is illustrated in Fig. 3 in the case of the cytozine nucleobase. The $G W_{p s c}$ calculations are found in particular to come in excellent agreement with a recent many-body wavefunction coupled-cluster equation-of-motion calculation [68].

We now proceed in more detail with two quantities much less commonly studied within many-body perturbation theory, and however of crucial importance for organic systems, namely the strength of the electronphonon coupling, and the charge-transfer excitation energies in donor/acceptor organic dyads.

\section{Electron-phonon coupling in the $C_{60}$ fullerene within $G W$}

Electron-phonon coupling in organic systems is a crucial aspect when it comes in particular to understand the mobility of free carriers. The coupling to molecular vibrations is indeed regarded as the main cause responsible for the poor conductivity of organic semiconductors [69], limitating dramatically the photocurrent. As 
such, the relaxation energy, $\lambda_{r e l}$, induced by the arrival of an electron (or hole) onto a molecule, has been the subject of many experimental and theoretical studies $[69,70]$. Such a relaxation energy can be obtained as the difference between the ground state energy of the charged system in its equilibrium geometry, and that of the charged system in its neutral equilibrium geometry. As such, DFT calculations are well justified, even though clear differences can be observed depending on the exchange-correlation functional adopted [71].

The same problem can also be considered by calculating individual electron-phonon matrix elements on the basis e.g. of perturbative DFT (DFPT) [72]. The calculation of the "scattering" matrix elements of electrons by vibrational modes lays the foundation of many fields in condensed matter physics, such as phononmediated superconductivity or inelastic scattering of free carriers in the normal state. Further, the relaxation energy can also be calculated as a sum over all phonon modes contribution, namely: $2 \lambda_{\text {rel }}=\sum_{\nu} V_{\nu}^{e p}$, with:

$V_{\nu}^{e p}=\frac{g_{\nu}}{M \omega_{\nu}^{2}} \sum_{i, j=1}^{g_{E_{F}}} \frac{\left|<\phi_{i}\right|\left(e_{\nu} \cdot \nabla\right) V^{S C F}\left|\phi_{j}>\right|^{2}}{g_{E_{F}}^{2}}$

where $\left(e_{\nu} \cdot \nabla\right) V^{S C F}$ is the normalized variation of the self-consistent potential under distortion of the molecule along the vibrational mode with index $(\nu)$, degeneracy $g_{\nu}$ and frequency $\omega_{\nu}$. The $(i, j)$ indices run over the electronic manifold at the Fermi level with degeneracy $g_{E_{F}}[73]$. We have used here the notation $V^{e p}$ traditionally used to label the attractive potential "glueing" electrons in Cooper pairs in the BCS theory of superconductivity. The above formula is the molecular limit $[77,78]$ of the central definition used in $a b$ initio studies of phonon-mediated superconductivity in extended solids.

Fullerenes are paradigmatic in both fields of superconductivity, through the well known case of the superconducting fullerides family [79-81], and also in organic photovoltaic systems since fullerene derivatives, such as $\mathrm{C}_{60}-\mathrm{PCMB}$, are the most widely used acceptors in organic cells. As such, the study of the relaxation energy, or equivalently of the $V_{\nu}^{e p}$ coupling energies, in the fullerenes, has recently regained much interest with several experimental $[83,82]$ and theoretical [51,84-86] studies. The $a b$ initio results are compiled in Table 2 for the $H_{g}$ and $A_{g}$ modes that couple to the three-fold $t_{1 u}$ lowest unoccupied (LUMO) manifold, of interest for superconducting alkali doped fullerides and for the propagation of electrons in acceptor fullerenes derivatives.

The calculated LDA electron-phonon coupling energies $[51,87]$ reported in Table 2, are significantly lower than the available experimental values extracted from

\begin{tabular}{c|c|c|c|c}
\hline Mode & LDA & Hybrids & $G W$ & Exp. \\
\hline$A_{g}(1)$ & 0.5 & $1.2-1.7$ & 1.0 & \\
$A_{g}(2)$ & 7.7 & $10.5-12.9$ & 15.0 & \\
$H_{g}(1)$ & 5.1 & $5.3-6.0$ & 6.4 & \\
$H_{g}(2)$ & 9.9 & $10.8-13.8$ & 11.2 & \\
$H_{g}(3)$ & 9.1 & $11.0-16.7$ & 13.9 & \\
$H_{g}(4)$ & 4.1 & $4.2-5.3$ & 5.6 & \\
$H_{g}(5)$ & 4.2 & $5.0-6.7$ & 5.2 & \\
$H_{g}(6)$ & 2.1 & $2.1-4.2$ & 2.3 & \\
$H_{g}(7)$ & 16.9 & $23.0-27.7$ & 27.6 & \\
$H_{g}(8)$ & 13.7 & $17.0-19.3$ & 20.4 & \\
\hline Sum $A_{g}$ & 8.2 & $11.7-14.6$ & 16.0 & $10.5(11)$ \\
Sum $H_{g}$ & 65.1 & $78.4-99.7$ & 92.5 & $96.2-96.5(147)$ \\
\hline Total & 73.3 & $90.1-114.3$ & 108.5 & $106.7(158)$ \\
\hline
\end{tabular}

Table 2 Calculated electron-phonon coupling contributions to $\mathrm{V}^{e p}$ (in $\mathrm{meV}$ ) for the $A_{g}$ and $H_{g}$ modes calculated within LDA and with various hybrid functionals. Hybrid functionals data from Refs. [84-86]), LDA and $G W_{p s c}$ from Ref. [51]. Experimental data range from the recent Refs. [86,82]. The older 1995 data by Gunnarsson and coworkers [88] are indicated in parenthesis.

photoemission (PES) experiments, with measured $V^{e p}$ potentials found to extend from $96 \mathrm{meV}$ to $147 \mathrm{meV}$ for the $H_{g}$ modes contribution [82,83,88], and from $107 \mathrm{meV}$ to $158 \mathrm{meV}$ including both $A_{g}$ and $H_{g}$ contributions $[83,88]$. By comparison, the use of hybrid functionals (column 4, Table 2), with an amount of exact exchange ranging from $20 \%$ [89] to $30 \%$, is shown to yield significantly larger values, with the coupling constants increasing with the percentage of exact exchange [84-86].

Even though in better agreement with experiment, the significant evolution of the coupling energies with the functional parameters clearly brings the standard question of knowing which functional should be used for a given system. This was recently emphasized in the case of graphene [90] where it was shown that LDA would dramatically underestimate the coupling energy, consistently with the present results, while on the contrary B3LYP [89] dramatically overestimates it.

To understand the failure of the standard DFT-LDA approach, and make the connection with $G W$ calculations, we note that in the case of finite size systems, the calculation of electron-phonon matrix elements can be very straightforwardly related, using the HelmannFeynman theorem, to the evolution of the electronic energy level (here the 3 -fold $t_{1 u}$ LUMO) with respect to the vibrational mode deformation, namely:

$$
\sum_{i, j=1}^{3}\left|<\phi_{i}\right|\left(e_{\nu} \cdot \nabla\right) V^{S C F}\left|\phi_{j}>\right|^{2}=\sum_{i=1}^{3}\left|\left(e_{\nu} \cdot \nabla\right) \varepsilon_{i}\right|^{2}
$$

Such an equation, that reformulates the electronphonon coupling problem in terms of the single-electron 
energy levels, clearly questions the standard DFT-LDA approach with Kohn-Sham eigenvalues known to be in severe disagreement with experiment for molecular systems. On the contrary, the quasiparticle $G W$ formalism, which provides much better quasiparticle energies, appears as a novel interesting framework for calculating electron-phonon matrix elements. DFT calculations are still central however by providing the vibrational phonon modes, a ground-state property.

The results of our $G W_{p s c}$ calculations [51] are compiled in the last but one column of Table 2. Clearly, the $G W_{p s c}$ electron-phonon coupling energies are significantly larger than the corresponding DFT-LDA values, and fall within the range obtained with hybrid functionals, but without any adjustable parameter. As compared to experiment, the $92.5 \mathrm{meV}$ total $V^{e p}$ potential energy for the $H_{g}$ modes is in good agreement with the two most recent $96.2 \mathrm{meV}$ and $96.5 \mathrm{meV}$ independent experimental estimates of Ref. [86] (Table V) and Ref. [82], respectively. Further, the total $G W_{p s c}$ coupling of $108.5 \mathrm{meV}$ is also in close agreement with the latest $106.7 \mathrm{meV}$ experimental value [83,86,91].

The present results clearly demonstrate that the $G W$ formalism, beyond its traditional use in band structure calculations, can also be of much interest for the calculation of electron-phonon coupling matrix elements, at least in the case of organic systems where DFTLDA Kohn-Sham eigenvalues are found to be dramatically inaccurate. While hybrid functional calculations may lead to accurate values, the case of graphene [90] shows that they can also lead to very bad results, underlying the importance of parameter-free $G W$ calculations known to perform equally well for extended and finite size systems. The generalization of the present $G W$ approach to extended systems stands however as a clear methodological challenge, in particular for nonzero phonon momentum q-vectors.

\section{Charge transfer excitations in donor/acceptor complexes}

In order to generate free charges that will participate to the photocurrent, the photoinduced electron-hole (excitonic) pairs must be dissociated [3]. Due to confinement and poor screening, the typical binding energy of excitons in standard organic cells is of the order of several hundreds of meV. This is significantly larger than in e.g. bulk silicon where the exciton binding energy is of the order of 10-20 meV. The dissociation in organic cells can nevertheless be achieved at a type-II donor/acceptor interface where the band offsets favour the "jump" of the electron from the donor side (e.g. P3HT polymers) to

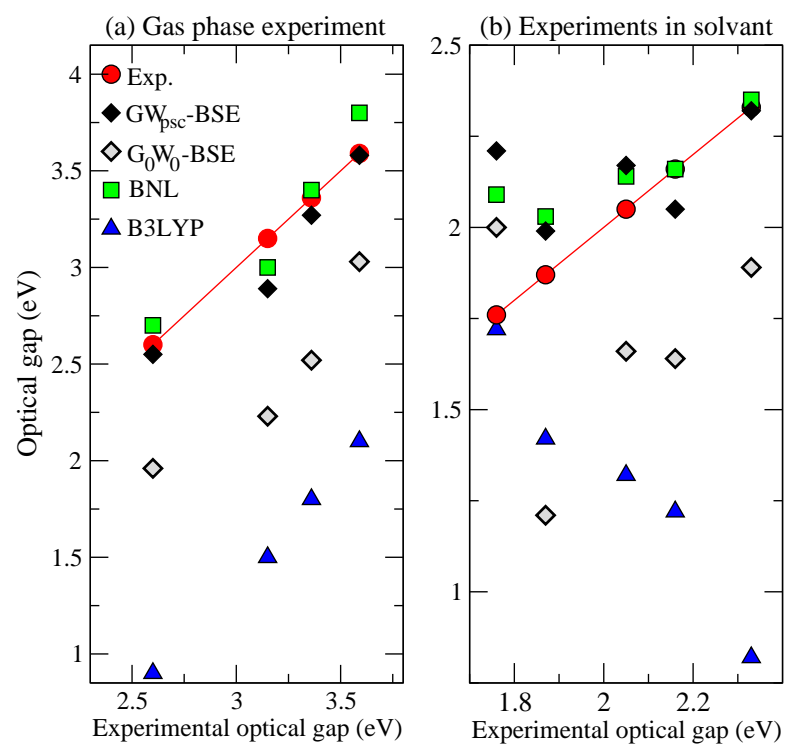

Fig. 4 (Color online) First singlet excitation energies in eV. Experimental data (red circles) are from (a) Ref. [95] for gas phase experiments on benzene, toluene, o-xylene and naphtalene donors, and (b) Ref. [96] for experiments in solution on anthracene and its derivatives. Following Ref. [94], a constant $0.32 \mathrm{eV}$ shift has been added to the experimental data in (b) to (tentatively) mimic the bathochromic shift. The B3LYP (blue triangle up) and BNL (green squares) data are the theoretical TDDFT results from Ref. [94]. The $G_{0} W_{0}-B S E$ (grey diamond) and $G W_{p s c}-B S E$ (black diamond) are the present FIESTA results. Figures (a) and (b) are not on the same energy scale.

the acceptor side (e.g. functionalized fullerenes), generating as an intermediate to dissociation a "chargetransfer" excited state with hole and electron weakly overlaping. The mechanisms driving such a dissociation, and the ways to improve it with respect to the adverse recombination, are still very badly understood $[2,3]$.

For reasons that have been hinted above, chargetransfer excitations are badly described within TDDFT if standard adiabatic (semi)local exchange-correlation kernels are used [92]. Charge-transfer excitations are indeed found to be located at much too small energy as compared to intramolecular excitations with holes and electrons presenting a strong spatial overlap. To cure such a problem, complex non-local kernels, within e.g. the so-called range-separated functionals family, have been implemented and tested, leading potentialy to excellent results. However, as shown e.g. in Ref. [93], the excitation energies strongly depend on the "localization" parameter chosen to tune the range of the interaction, leading again to the problem of determining $a b$ initio the correct parameter for a given finite or extended system. 
Recently, a scheme proposing to adjust such a parameter to reproduce the photoemission band gap as obtained ab initio from $\triangle \mathrm{SCF}$ DFT calculations was shown to lead to excellent charge-transfer excitations without any a priori knowledge of the experimental data [94]. Such a scheme was tested on several donor/acce organic systems composed of a TCNE (tetracyanoethylene) acceptor combined with benzene, naphtalene and anthracene derivatives, systems for which gaz phase [95] and solvated experiments [96] are available. A plot of the HOMO and LUMO eigenstates (DFT-LDA calculations at the TZ2P level) was given in Fig. 1 in the case of the TCNE with 9-methyl-anthracene complex, showing the charge transfer character of the HOMO to LUMO excitation. As shown in Fig. 4 under the label BNL (green squares), the resulting lowest excitation energies, which are charge transfer excitations, are in much better agreement with experiment (red circles) than standard TDDFT calculations with the B3LYP kernel (blue triangles). The availability of precise experimental data and state-of-the-art TDDFT calculations offers a precious mean to benchmark $G W$-BSE calculations on such charge-transfer systems. This was achieved in Refs. [37,47].

The charge-transfer excitations obtained within the BSE formalism as implemented in the FIESTA code [47], with a TZ2P basis description of the input LDA KohnSham states, are represented in Fig. 4 (grey and black diamonds) and compiled in Table 3. As emphasized above, the quasiparticle band gap obtained from singleshot $G_{0} W_{0}$ calculations starting from LDA eigenstates is significantly too small, yielding too small BSE excitation energies (grey diamonds). When the BSE calculations are preceeded by a (partial) self-consistent $G W_{p s c}$ calculation with update of the eigenvalues, the resulting excitation energies (black diamonds) are found to be in excellent agreement with experiment, with a mean absolute error of the order of $0.1 \mathrm{eV}$. Our calculations for benzene derivatives are found further to be in excellent agreement with planewave calculations using the GW/BSE Yambo code $[37,47,97]$ (see numbers in parenthesis in Table 3), demonstrating the reliability of the present Gaussian-basis implementation.

\section{Conclusions}

The present studies, together with the various recent attempts from the community to study organic systems within the $G W$ and BSE many-body perturbation theories, clearly show that excellent results can be obtained for quasiparticle properties, electron-phonon coupling constants, and charge-transfer excitations. This requests however that, beyond the standard non-self-consistent

\begin{tabular}{cccc}
\hline \hline donor & $G_{0} W_{0}-B S E$ & $G W_{p s c}$-BSE & Exp. \\
\hline benzene & $3.03(3.10)$ & $3.58(3.63)$ & 3.59 \\
toluene & $2.52(2.67)$ & $3.27(3.37)$ & 3.36 \\
o-xylene & $2.23(2.28)$ & $2.89(3.00)$ & 3.15 \\
naphtalene & 1.96 & 2.55 & 2.60 \\
\hline MAE & $0.74(0.68)$ & $0.10(0.07)$ & - \\
\hline \hline or substituent & \multicolumn{4}{c}{ anthracene derivatives } \\
\hline none & 1.66 & 2.17 & $(2.05)$ \\
9-carbo-methoxy & 1.64 & 2.05 & $(2.16)$ \\
9-cyano & 1.89 & 2.32 & $(2.33)$ \\
9-methyl & 1.21 & 1.99 & $(1.87)$ \\
9,10-dimethyl & 2.00 & 2.21 & $(1.76)$ \\
9-formyl & 1.89 & 2.32 & $(2.22)$ \\
\hline MAE & 0.43 & 0.15 & - \\
\hline \hline
\end{tabular}

Table 3 Experimental and theoretical optical gap (eV) for donor-TCNE complexes. Theoretical results in parenthesis are obtained with the Yambo package. MAE is the mean absolute error. Experimental results are from Ref. [95,96]. The experimental results in parenthesis were obtained in solution and a $0.32 \mathrm{eV}$ constant energy has been added following Stein and coworker [94] to account for solvation effects.

$G_{0} W_{0}$ approach based on LDA eigenstates, we use either self-consistency in some form, or a different zeroth order starting point.

The two topics discussed in more details in the present review, namely the calculation of the electron-phonon effective coupling potential, and the energy of the chargetransfer excitations, are certainly of much importance in the field of organic photovoltaics and beyond. In particular, the difficulties encountered within the TDDFT framework to treat non-local excitations clearly paves the way for the use of the Bethe-Salpeter equations that treat on the same footing local and non-local excitations thanks to the screened Coulomb potential $W\left(\mathbf{r}, \mathbf{r}^{\prime}\right)$. The success of the BSE formalism in addressing both intramolecular and charge-transfer excitations can be compared to its ability to treat both extended (Wannier) and localized (Frenkel) excitons in extended semiconductors or insulators. Concerning the electron-phonon coupling, the very widespread use of density functional perturbative theory to study in particular BCS superconductors, and the present results emphasizing the possible failure of DFT-LDA calculations, strongly call for a careful exploration of the difference between LDA and $G W$-based electron-phonon coupling constants in covalent or metallic extended systems.

It would certainly be unwise to conclude at that stage that the $G W$ and BSE approaches offer a better compromise between CPU time and accuracy than the specific many-body variational or perturbative theories developed in the quantum chemistry community for finite size molecular systems. A significant advantage of the GW and BSE approaches however is that they have been developed for extended systems. As a 
matter of fact, the success of the GW approximation certainly relies on the strength of the screening in extended systems that considerably reduces the effective Coulomb potential. As such, in actual organic photovoltaic cells, namely when organic molecules are not isolated, but surrounded by a screening environment, there is much confidence that the GW and BSE approaches will keep being extremely efficient. Such an observation points clearly in the direction of an important challenge in the field of many-body perturbation theory applied to organic systems, namely its ability to tackle complex non-periodic and disordered systems such as realistic donor/acceptor interfaces [98].

Acknowledgements Computing time has been provided by the local CIMENT and national IDRIS supercomputing (Project No. 100063) centers in Grenoble and Orsay, respectively. The authors acknowledge Dr. Laflamme Janssen, Pr. Michel Côté, and Pr. Erich Runge, with whom parts of the work presented in this review were achieved, and Pr. Mark Casida for useful discussions.

\section{References}

1. Organic Photovoltaics: Concepts and Realization, Springer Series in Material Science, edited by C. Brabec, V. Dyakonov, J. Parisi, and N. S. Sariciftci (Springer, New York, 2003).

2. Bernard Kippelen and Jean-Luc Brédas, Organic photovoltaics, Energy Environ. Sci. 2, 251-261 (2009).

3. J.L. Brédas, J.E.Norton, J. Cornil et al., Molecular Understanding of Organic Solar Cells: The Challenges, Accounts of Chemical Research 42, 1691 (2009).

4. Quantitative information on solar energy can be found at the International Energy Agency Photovoltaic Power Systems Program at www.iea-pvps.org.

5. S. Park, A. Boy, S. Beaupré, S. Cho et al., Bulk heterojunction solar cells with internal quantum efficiency approaching 100\%, Nat. Photonics 3, 297-302 (2009).

6. H-Y. Chen et al., Polymer solar cells with enhanced opencircuit voltage and efficiency, Nat. Photonics 3, 649 (2009).

7. C.J. Brabec et al., Origin of the open circuit voltage of plastic solar cells, Adv. Funct. Mater. 11, 374-380 (2001).

8. M.C. Scharber et al., Design rules for donors in bulkheterojunction solar cells - Towards $10 \%$ energy-conversion efficiency, Adv. Mater. 18, 789 (2006).

9. F.B. Kooistra et al., Increasing the open circuit voltage of bulk heterojunction solar cells by raising the LUMO level of the acceptor, Org. Lett. 9, 551-554 (2007).

10. A. Tkatchenko, L. Romaner, O.T. Hofmann et al., Van der Waals Interactions Between Organic Adsorbates and at Organic/Inorganic Interfaces, MRS Bull. 35, Issue 6, 435442 (2010).

11. W.G. Aulbur, L. Jonsson, J.W. Wilkins, Quasiparticle calculations in solids, Solid State Physics: Advances in reserach and applications, Vol. 54, pp. 1-218 (2000).

12. M. K. Shukla and J. Leszczynski, A density functional theory study on the effect of shape and size on the ionization potential and electron affinity of different carbon nanostructures, Chem. Phys. Lett. 428, 317 (2006).

13. Z. Zhang et al., J. Phys. Chem. C 112, 19158 (2008).
14. From the NIST chemistry webbook at http://webbook.nist.gov/chemistry/.

15. S. Refaely-Abramson, R. Baer, L. Kronik, Fundamental and excitation gaps in molecules of relevance for organic photovoltaics from an optimally tuned range-separated hybrid functional Phys. Rev. B 84, 075144 (2011).

16. I. Dabo, A. Ferretti, N. Pilvert, et al., Koopmans' condition for density-functional theory, Phys. Rev. B 82, 115121 (2010).

17. L. Hedin, New Method for Calculating the One-Particle Green's Function with Application to the Electron-Gas Problem, Phys. Rev. 139, A796 (1965).

18. G. Strinati, H.J. Mattausch, W. Hanke, Dynamical Correlation Effects on the Quasiparticle Bloch States of a Covalent Crystal, Phys. Rev. Lett. 45, 290 (1980); ibid, Dynamical aspects of correlation corrections in a covalent crystal, Phys. Rev. B 25, 2867 (1982).

19. M.S. Hybertsen and S.G. Louie, Electron correlation in semiconductors and insulators: Band gaps and quasiparticle energies, Phys. Rev. B 34, 5390 (1986).

20. R.W. Godby, M. Schlüter, and L.J. Sham, Self-energy operators and exchange-correlation potentials in semiconductors, Phys. Rev. B 37, 10159 (1988).

21. L. J. Sham and T. M. Rice, Many-Particle Derivation of the Effective-Mass Equation for the Wannier Exciton, Phys. Rev. 144, 708 (1966); W. Hanke and L. J. Sham, Many-Particle Effects in the Optical Excitations of a Semiconductor, Phys. Rev. Lett. 43, 387 (1979).

22. G. Strinati, Dynamical Shift and Broadening of Core Excitons in Semiconductors, Phys. Rev. Lett. 49, 1519 (1982).

23. Rohlfing and S. G. Louie, Excitonic Effects and the Optical Absorption Spectrum of Hydrogenated Si Clusters, Phys. Rev. Lett. 80, 3320 (1998).

24. L.X. Benedict, E. Shirley, and R. B. Bohn, Optical Absorption of Insulators and the Electron-Hole Interaction: An Ab Initio Calculation, Phys. Rev. Lett. 80, 4514 (1998).

25. S. Albrecht, L. Reining, R. Del Sole, and G. Onida, $A b$ Initio Calculation of Excitonic Effects in the Optical Spectra of Semiconductors, Phys. Rev. Lett. 80, 4510 (1998).

26. M. L. Tiago, J. E. Northrup, and S. G. Louie, Ab initio calculation of the electronic and optical properties of solid pentacene, Phys. Rev. B 67, 115212 (2003).

27. K. Hummer, P. Puschnig, C. Ambrosch-Draxl, Lowest optical excitations in molecular crystals: Bound excitons versus free electron-hole pairs in anthracene, Phys. Rev. Lett. 92, 147402 (2004).

28. P. Puschnig and C. Ambrosch-Draxl, Excitons in organic semiconductors, Com. Ren. Phys. 10, 504-513 (2009).

29. N. Dori, M. Menon, L. Kilia et al., Valence electronic structure of gas-phase 3,4,9,10-perylene tetracarboxylic acid dianhydride: Experiment and theory, Phys. Rev. B 73, 195208 (2006).

30. E.C. Ethridge, J.L. Fry, M. Zaider, Quasiparticle spectra of trans-polyacetylene, Phys. Rev. B 53, 3662 (1996).

31. J.-W. van der Horst, P.A. Bobbert, and M.A.J. Michels, G. Brocks, P.J. Kelly, Ab Initio Calculation of the Electronic and Optical Excitations in Polythiophene: Effects of Intra- and Interchain Screening, Phys. Rev. Lett. 83, 4413 (1999),

32. Michael Rohlfing and Steven G. Louie, Optical Excitations in Conjugated Polymers, Phys. Rev. Lett. 82, 1959 (1999).

33. M.L. Tiago, M. Rohlfing, S.G. Louie, Bound excitons and optical properties of bulk trans-polyacetylene, Phys. Rev. B 70, 193204 (2004).

34. G.M. Rignanese, X. Blase, S.G. Louie, Quasiparticle effects on tunneling currents: A study of $\mathrm{C}(2) \mathrm{H}(4)$ adsorbed 
on the $\mathrm{Si}(001)-(2 \times 1)$ surface Phys. Rev. Lett. 86, 2110 (2001).

35. J. B. Neaton, Mark S. Hybertsen, and Steven G. Louie, Renormalization of Molecular Electronic Levels at MetalMolecule Interfaces, Phys. Rev. Lett. 97, 216405 (2006).

36. S.Y. Quek, J.B. Neaton, M.S. Hybertsen, E. Kaxiras, S.G. Louie, Negative differential resistance in transport through organic molecules on silicon Phys. Rev. Lett. 98, 066807 (2007).

37. J.M. Garcia-Lastra and K.S. Thygesen, Renormalization of Optical Excitations in Molecules near a Metal Surface, Phys. Rev. Lett. 106, 187402 (2011); ibid, Erratum, Phys. Rev. Lett. 107, 179901 (2011).

38. I. Tamblyn, P. Darancet, S.Y. Quek, S.A.Bonev, J.B. Neaton, Electronic energy level alignment at metalmolecule interfaces with a GW approach Phys. Rev. B 84, 201402(R) (2011).

39. M. Strange, C. Rostgaard, H. Hakkinen, K.S. Thygesen, Self-consistent GW calculations of electronic transport in thiol- and amine-linked molecular junctions Phys. Rev. B 83, 115108 (2011).

40. T. Rangel, A. Ferretti, P.E. Trevisanutto, V. Olevano, and G.-M. Rignanese, Transport properties of molecular junctions from many-body perturbation theory, Phys. Rev. B 84, 045426 (2011).

41. M.L. Tiago and J.R. Chelikowsky, First-principles GWBSE excitations in organic molecules, Sol. State Comm. 136, 333-337 (2005).

42. M. Palummo, C. Hogan, F. Sottile et al., Ab initio electronic and optical spectra of free-base porphyrins: The role of electronic correlationr, J. Chem. Phys. 131, 084102 (2009).

43. Y. Ma, M. Rohlfing, C. Molteni, Excited states of biological chromophores studied using many-body perturbation theory: Effects of resonant-antiresonant coupling and dynamical screening, Phys. Rev. B 80, 241405 (2009).

44. G. Stenuit, C. Castellarin-Cudia, O. Plekan et al., Valence electronic properties of porphyrin derivatives, Phys. Chem. Chem. Phys. 12, 10812-10817 (2010).

45. P. Umari, G. Stenuit, S. Baroni, GW quasiparticle spectra from occupied states only, Phys. Rev. B 81, 115104 (2010).

46. X. Blase, C. Attaccalite, V. Olevano, First-principles GW calculations for fullerenes, porphyrins, phthalocyanine, and other molecules of interest for organic photovoltaic applications, Phys. Rev. B 83, 115103 (2011).

47. X. Blase and C. Attaccalite, Charge-transfer excitations in molecular donor-acceptor complexes within the many-body Bethe-Salpeter approach, Appl. Phys. Lett. 99, 171909 (2011).

48. D. Foerster, P. Koval, D. Sánchez-Portal, An O(N(3)) implementation of Hedin's GW approximation for molecules., J. Chem. Phys. 135, 074105 (2011).

49. M. Noa, R. Xinguo, J.E. Moussa et al., Electronic structure of copper phthalocyanine from $\mathrm{G}(0) \mathrm{W}(0)$ calculations, Phys. Rev. B 84, 195143 (2011).

50. C. Faber, C. Attaccalite, V. Olevano, E. Runge, X. Blase, First-principles GW calculations for DNA and RNA nucleobases, Phys. Rev. B 83, 115103 (2011).

51. C. Faber, J. Jonathan Laflamme, M. Côté, E. Runge, $\mathrm{X}$. Blase, Electron-phonon coupling in the $\mathrm{C}(60)$ fullerene within the many-body GW approach, Phys. Rev. B 84, 155104 (2011).

52. This code originates in a previous implementation of TDDFT using the strictly-localized atomic-like orbital basis and states implemented in the DFT SIESTA [53] package.
See: X. Blase and P. Ordejón, Dynamical screening and absorption within a strictly localized basis implementation of time-dependent LDA: From small clusters and molecules to aza-fullerenes, Phys. Rev. B 69, 085111 (2004).

53. E. Artacho, E. Anglada, O. Dieguez, J. D. Gale, A. Garca, J. Junquera, R. M. Martin, P. Ordejón, J. M. Pruneda, D. Sánchez-Portal and J. M. Soler, The SIESTA method; developments and applicability, J. Phys.: Condens. Matter 20, 064208 (2008).

54. E. Runge and E. K. U. Gross, Phys. Rev. Lett. 52, 997 (1984); Time-Dependent Density Functional Theory, edited by M. A. L. Marques, C. A. Ullrich, F. Nogueira, A. Rubio, K. Burke, and E. K. U. Gross (Springer, Berlin Heidelberg 2006).

55. M.E. Casida, Review: Time-Dependent DensityFunctional Theory for Molecules and Molecular Solids, J. Mol. Struct. (Theochem) 914, 3 (2009).

56. Michael Rohlfing, Peter Krüger, and Johannes Pollmann, Efficient scheme for GW quasiparticle band-structure calculations with applications to bulk $\mathrm{Si}$ and to the $\mathrm{Si}(001)-(21)$ surface, Phys. Rev. B 52, 1905 (1995).

57. Y. Pavlyukh, W. Hubner, Lifetime of quasiparticle states in metallic clusters from GW theory, Phys. Lett. A 327, 241 (2004).

58. Eric Chang, Giovanni Bussi, Alice Ruini, and Elisa Molinari, Excitons in Carbon Nanotubes: An Ab Initio Symmetry-Based Approach, Phys. Rev. Lett. 92, 196401 (2004).

59. I. Cherkes, S. Klaiman, and N. Miseyev, Spanning the Hilbert space with an even tempered Gaussian basis set Int. J. Quantum Chem. 109, 2996 (2009).

60. F. Aryasetiawan, O. Gunnarsson, Product-basis method for calculating dielectric matrices, Phys. Rev. B 49, 16214 (1994).

61. M.L. Tiago, J.R. Chelikowsky, Optical excitations in organic molecules, clusters, and defects studied by firstprinciples Green's function methods, Phys. Rev. B 73, 205334 (2006).

62. E. Anglada, J. M. Soler, J. Junquera and E. Artacho, Systematic generation of finite-range atomic basis sets for linear-scaling calculations, Phys. Rev. B 66, 205101 (2002).

63. B. Farid, in Electron Correlation in the Solid State, edited by N. H. March (World Scientific, Singapore, 1999), p. 217, and references therein.

64. P. H. Hahn, W. G. Schmidt, and F. Bechstedt, Molecular electronic excitations calculated from a solid-state approach: Methodology and numerics, Phys. Rev. B 72, 245425 (2005).

65. C. Rostgaard, K.W. Jacobsen, and K. S. Thygesen, Fully self-consistent GW calculations for molecules, Phys. Rev. B 81, 085103 (2010).

66. K. Kaasbjerg and K. S. Thygesen, Benchmarking GW against exact diagonalization for semiempirical models, Phys. Rev. B 81, 085102 (2010).

67. S.H. Ke, All-electron GW methods implemented in molecular orbital space: Ionization energy and electron affinity of conjugated molecules, Phys. Rev. B 84, 205415 (2011).

68. K.B. Bravaya, O. Kostko, S. Dolgikh, A. Landau et al., J. Phys. Chem. A 114, 12305-12317 (2010); and references therein.

69. V. Coropceanu, J. Cornil, D. A. da Silva Filho, Y. Olivier, Y., Silbey, R. and Brédas, J.-L. Charge Transport in Organic Semiconductors. Chem. Rev. 107, 926-952 (2007)

70. Richard C. Hatch, David L. Huber, and Hartmut Hochst, Electron-Phonon Coupling in Crystalline Pentacene Films, Phys. Rev. Lett. 104, 047601 (2010). 
71. In the case of acenes, see: Assessment of densityfunctional models for organic molecular semiconductors: The role of Hartree-Fock exchange in charge-transfer processes, J.C. Sancho-García, Chemical Physics 331, 321-331 (2007).

72. Stefano Baroni, Stefano de Gironcoli, Andrea Dal Corso, and Paolo Giannozzi, Phonons and related crystal properties from density-functional perturbation theory, Rev. Mod. Phys. 73, 515 (2001).

73. The equivalence between the approach based on differences of total energies, and the one involving individual electron-phonon matrix elements, (compare e.g. formulas (1) and (4) in Ref. [74]) has been validated numerically for many systems within DFT (see e.g. in the case of the acenes the results of Refs. [74] and [75]), even though formally the only existing demonstrations assume the coupling between vibrational modes and free electrons at the Fermi level within the so-called independent bozon model [76], one of the few exactly solvable many-body problem.

74. V. Coropceanu, M. Malagoli, D. A. da Silva Filho, N. E. Gruhn, T.G. Bill, and J. L. Brédas Hole- and ElectronVibrational Couplings in Oligoacene Crystals: Intramolecular Contributions, Phys. Rev. Lett. 89, 275503 (2002).

75. T. Kato et al., Electron-phonon coupling in negatively charged acene- and phenanthrene-edge-type hydrocarbon crystals, J. Chem. Phys. 116, 3420 (2002).

76. Gerald D. Mahan, Many-Particle Physics, Plenum Press (New York and London), 2nd Eds (1990).

77. M. Schlüter, M. Lannoo, M. Needels, G.A. Baraff, and D. Tománek, Electron-phonon coupling and superconductivity in alkali-intercalated $C 60$ solid, Phys. Rev. Lett. 68, 526 (1992).

78. V.P. Antropov, O. Gunnarsson, and A.I. Liechtenstein, Phonons, electron-phonon, and electron-plasmon coupling in $C_{60}$ compounds Phys. Rev. B 48, 7651 (1993).

79. A.F. Hebard, M.J. Rosseinsky, R.C. Haddon, D.W. Murphy et al., Superconductivity at $18 \mathrm{~K}$ in potassium-doped $C_{60}$, Nature (London) 350, 600 (1991).

80. For a review, see: O. Gunnarsson, Superconductivity in fullerides, Rev. Mod. Phys. 69, 575-606 (1997).

81. A.Y. Ganin, Y. Takabayashi, Y.Z. Khimyak, S. Margadonna, A. Tamai, M.J. Rosseinsky, K. Prassides, Bulk superconductivity at $38 \mathrm{~K}$ in a molecular system, Nature Mater. 7, 367 (2008).

82. I.D. Hands, J.L. Dunn, L. Janette, C.A. Bates, A. Colin, M.J. Hope, J. Michael, S.R. Meech, D.L. Andrews, Vibronic interactions in the visible and near-infrared spectra of $C_{60}^{-}$ anions, Phys. Rev. B 77, 115445 (2008).

83. X.-B. Wang, H.-K. Woo, and L.-S. Wang, Vibrational cooling in a cold ion trap: Vibrationally resolved photoelectron spectroscopy of cold $C 60^{-}$anions, J. Chem. Phys. 123, 051106 (2005).

84. M. Saito, Electron-phonon coupling of electron- or holeinjected $C_{60}$, Phys. Rev. B 65, 220508(R) (2002).

85. J. Laflamme Janssen, M. Côté, S.G. Louie, M.L. Cohen, Electron-phonon coupling in $C_{60}$ using hybrid functionals, Phys. Rev. B 81, 073106 (2010).

86. N. Iwahara, T. Sato, K. Tanaka, and L.F. Chibotaru, Vibronic coupling in $C_{60}^{-}$anion revisited: Derivations from photoelectron spectra and DFT calculations, Phys. Rev. B 82, 245409 (2010).

87. Very similar results are obtained at the PBE level. See Ref. [85].

88. O. Gunnarsson, H. Handschuh, P.S. Bechthold, B. Kessler, G. Gantefor, W. Eberhardt, Photoemission Spectra of $C_{60}^{-}$: Electron-Phonon Coupling, Jahn-Teller Effect, and Superconductivity in the Fullerides, Phys. Rev. Lett. 74, 1875 (1995).

89. The lowest $20 \%$ percentage of exact exchange corresponds to the well-known B3LYP functional. See: A.D. Becke, Density-functional thermochemistry. III. The role of exact exchange, J. Chem. Phys. 98, 5648 (1993).

90. M. Lazzeri, C. Attaccalite, L. Wirtz, and F. Mauri, Impact of the electron-electron correlation on phonon dispersion: Failure of LDA and GGA DFT functionals in graphene and graphite, Phys. Rev. B 78, 081406 (2008).

91. An interesting observation is that a non-self-consistent $G_{0} W_{0}$ calculation starting from LDA eigenstates leads to a coupling constant which is still significantly larger than the DFT-LDA value, but smaller than the $G W_{p s c}$ one, and very similar to that of the hybrid B3LYP functional. As emphasized in the introductory section, single-shot $G_{0} W_{0}$ calculations result in too small band gaps, inducing overscreening expected to soften the variations of the ionic and electronic potential seen by the electrons upon lattice distortion. See data in Ref. [51].

92. Andreas Dreuw and Martin Head-Gordon, Failure of Time-Dependent Density Functional Theory for Long-Range Charge-Transfer Excited States: The Zincbacteriochlorin- Bacteriochlorin and Bacteriochlorophyll- Spheroidene Complexes, J. Am. Chem. Soc. 126, 4007 (2004).

93. Adrian W. Lange, and John M. Herbert, Both Intraand Interstrand Charge-Transfer Excited States in Aqueous B-DNA Are Present at Energies Comparable To, or Just Above, the $\pi-\pi^{*}$ Excitonic Bright States, J. Am. Chem. Soc. 131 , 3913-3922 (2009). See in particular the Supplementary materials.

94. T. Stein, L. Kronik, R. Baer, Reliable Prediction of Charge Transfer Excitations in Molecular Complexes Using Time-Dependent Density Functional Theory, J. Am. Chem. Soc. 131, 2818 (2009).

95. I.J. Hanazaki, Vapor-phase electron donor-acceptor tetracyanoethylene adn of sulfur-dioxide, Phys. Chem. 76, 1982 (1972).

96. J.M. Massnovi, E.A. Seddon, J.J. Kochi, Electrontransfer from anthracenes - Comparison of photoionization, charge-transfer excitation and electrochemical oxydation, J. Can, J. Chem. 62, 2552 (1984).

97. A. Marini, C. Hogan, M. Grüning, D. Varsano, Yambo: an $a b$ initio tool for excited state calculations, Comput. Phys. Commun. 180, 1392 (2009).

98. D.P. McMahon, D.L. Cheung, A. Troisi, Why Holes and Electrons Separate So Well in Polymer/Fullerene Photovoltaic Cells, J. Phys. Chem. Lett. 2, 2737-2741 (2011). 